\title{
Manipulation of single sub-femtolitre droplets via partial coalescence in a direct-current electric field
}

\author{
Mostafa Shojaeian ${ }^{1}$ and Steffen $\operatorname{Hardt}^{1, *}$ (D) \\ ${ }^{1}$ Fachbereich Maschinenbau, Fachgebiet Nano- und Mikrofluidik, Technische Universität Darmstadt, 64287 Darmstadt, Germany \\ *Corresponding author. E-mail: hardt@nmf.tu-darmstadt.de
}

Received: 8 July 2021; Revised: 10 September 2021; Accepted: 18 September 2021

Keywords: Droplet; Partial coalescence; Electrohydrodynamics; Droplet manipulation; Liquid sampling

\begin{abstract}
It is demonstrated how aqueous droplets with volumes down to the sub-femtolitre range can be manipulated, including the withdrawal of minute samples from the droplets. The underlying principle is that of partial coalescence with a liquid reservoir in an applied electric field. Upon partial coalescence, a droplet merges with a reservoir and reappears with a smaller diameter. The droplets studied here perform a reciprocating motion between two reservoirs during which their volume gets reduced. Manipulation of droplets with diameters down to $400 \mathrm{~nm}$ is reported. A similarity relation is derived expressing the ratio of droplet diameters before and after partial coalescence as a function of the ratio between electric and interfacial-tension forces. The presented scheme allows the withdrawal of minute samples from small droplets and could prove helpful in various applications where droplets are used as tiny reaction spaces or when the goal is to tailor the size of individual droplets.
\end{abstract}

\section{Impact Statement}

Droplet microfluidics has become a versatile technology with a multitude of applications. However, adding/withdrawing liquid into/from droplets with femtolitre volumes remains a challenge. The process reported here offers a solution to the liquid sampling problem and is controllable via the applied electric field. It could prove helpful in various applications where droplets are used as tiny reaction spaces or when the goal is to tailor the size of individual droplets.

\section{Introduction}

Droplets with diameters of the order of $1 \mu \mathrm{m}$ or below are of interest for a plethora of applications. One application with rapidly growing perspectives is to use droplets or vesicles as biochemical reaction spaces, mimicking the processes inside biological cells or unicellular organisms (Beneyton et al., 2018; dos Santos et al., 2020; Fanalista et al., 2019; Gach, Iwai, Kim, Hillson, \& Singh, 2017; Göpfrich, Platzman, \& Spatz, 2018; Haller et al., 2018; Shojaeian, Lehr, Göringer, \& Hardt, 2019; Van Nies et al., 2018; Wang, Du, Wang, Mu, \& Han, 2020; Weiss et al., 2018). For such applications, it is of key importance to be able to manipulate/control the microscopic reaction spaces and to analyse/monitor the processes occurring inside. One essential building block of manipulation/control strategies is to add liquid or dissolved species to the reaction spaces or to withdraw them from there. Such operations 
were achieved via merging of a droplet with a liquid meniscus (Song, Li, Munson, Ha, \& Ismagilov, 2006), electrohydrodynamic injection/extraction (Abate, Hung, Marya, Agresti, \& Weitz, 2010; Zeng, Pan, Zhang, Lin, \& Qin, 2011) or diffusive transport between a droplet and a reservoir (Shojaeian \& Hardt, 2020). Usually these operations become increasingly difficult the smaller the droplets get. With respect to analysing/monitoring processes inside tiny reaction compartments, cyclic voltammetry (Batchelor-McAuley et al., 2016), plasmon-based electrochemical current monitoring of redox reactions (Wang et al., 2015) and monitoring of electrochemical reactions using ultramicroelectrodes (Kim, Kim, \& Bard, 2015) were demonstrated, all inside single sub-femtolitre droplets. To produce reaction compartments, especially droplets, with diameters of the order of $1 \mu \mathrm{m}$ or below, a number of methods are available. Classical methods such as ultrasonication (Delmas et al., 2011) or high-pressure homogenization (Håkansson, 2019) usually yield quite broad droplet-size distributions. To better control the size of very small droplets, micro- or nanofluidic principles can be employed. Examples are membrane emulsification (Vladisavljević, 2019), droplet production at submicron-scale T-junctions (Toprakcioglu, Challa, Morse, \& Knowles, 2020), step emulsification (Malloggi et al., 2010) or tip streaming (Anna \& Mayer, 2006; Marín, Campo-Cortés, \& Gordillo, 2009; Suryo \& Basaran, 2006).

In this work, we present a new method to manipulate droplets with volumes on the sub-femtolitre scale. The method is based on the partial coalescence of aqueous droplets with an aqueous reservoir under a direct-current (DC) electric field. Already in the 1960s it was discovered (Allan \& Mason, 1961; Charles \& Mason, 1960a, 1960b) that, when an aqueous droplet is driven to an aqueous/oil interface by a DC electric field, it may rebound (non-coalescence) or re-emerge with a smaller size (partial coalescence). The non-coalescence mode was first rationalized in 2009 (Ristenpart et al., 2009). Driven by the electric field, a Taylor cone (Fernández de la Mora, 2007) forms at the droplet, after which a tiny liquid bridge is created between the droplet and the reservoir. For strong enough electric fields, the pressure inside the bridge exceeds the pressure in the droplet, thus driving liquid out of the bridge and causing pinch-off. As a result, the droplet does not coalesce with the reservoir, but bounces back, while at the same time its charge becomes inverted. In 2012, the partial coalescence mode between aqueous droplets and aqueous reservoirs was rationalized (Hamlin et al., 2012). For low enough values of the electric conductivity inside the drop, i.e. low enough values of the salt concentration, the droplet that re-emerges after a droplet comes into contact with the aqueous reservoir has a reduced volume.

Based on this preliminary work, we have demonstrated a device with which it is possible to transfer dissolved species between a reservoir and a droplet in a well-controlled manner (Shojaeian \& Hardt, 2020). In this device, a droplet reciprocates between two aqueous reservoirs, driven by a DC electric field. Through the liquid bridge forming upon non-coalescence, the droplet exchanges dissolved species with the reservoirs. Specifically, this means that, when the droplet serves as a tiny reaction space, it can work as a semi-batch reactor, where reagents are introduced from one reservoir and reaction products are withdrawn from the other reservoir.

In this work we demonstrate another mode of manipulating droplets with volumes down to the subfemtolitre range, which we believe is unprecedented for droplets in this size range. We show that, making use of partial coalescence, a part of the droplet liquid can be subtracted, even for droplets with diameters below $1 \mu \mathrm{m}$. This way, sampling of liquid from tiny reaction spaces is enabled. In context with synthetic cells that serve as a motivation for this work, this would mean that sampling operations become possible, analogous to the recently developed methods for extracting the contents of living cells (Actis, 2018).

\section{Experimental}

The experiments were conducted in a microfluidic device, shown in Figure 1. The design of this device is the same as previously described (Shojaeian \& Hardt, 2020). In brief, the key part of the chip is a cross-junction configuration. The shallow main channel is placed between two side channels of larger depth. The main channel contains the oil phase, and the side channels are filled with aqueous phase. The inlet sections of the side channels are connected to two ports of a power supply through needles, through which the DC electric field is applied. The width of the two side channels is different, so 


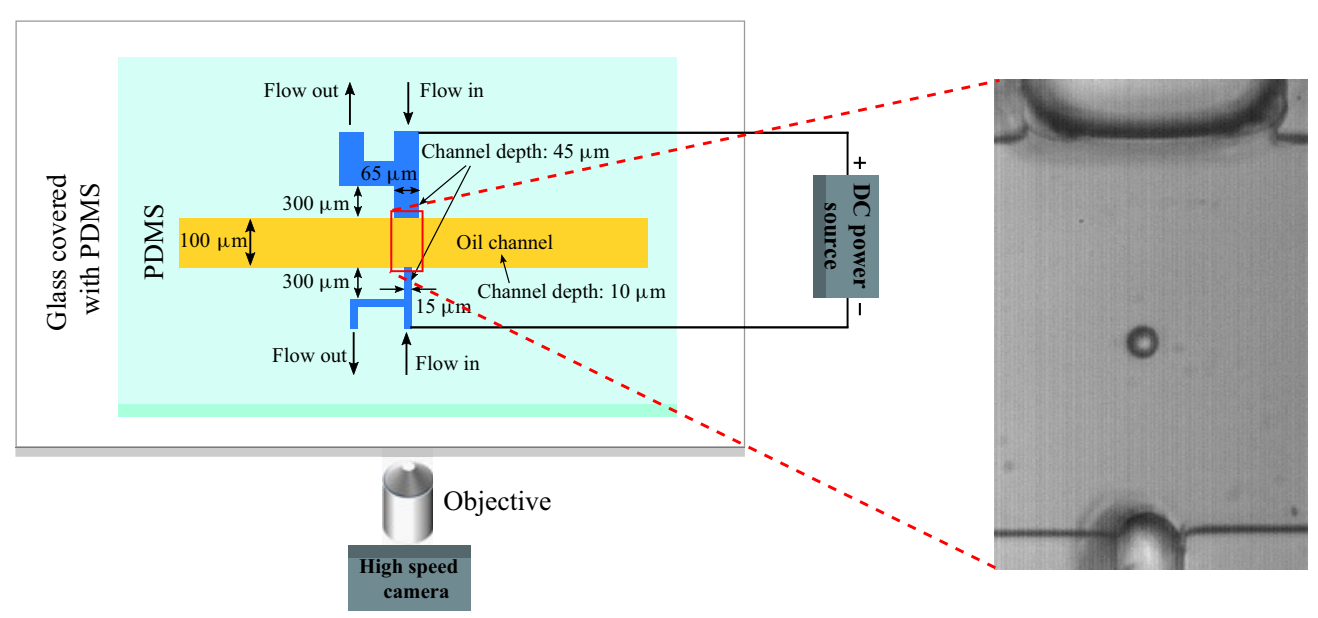

Figure 1. Schematic of the microfluidic device, together with the high-voltage source and the imaging equipment. The blow-up shows the region of interest (indicated as a red rectangle) with a droplet exposed to the electric field applied through the side channels.

that the electric field lines become concentrated at the side channel of smaller width. This asymmetry enables droplet production through electric field pulses from the side channel of smaller width. Time lapse images showing the droplet production are shown in the supplementary materials are available at https://doi.org/10.1017/flo.2021.12.

The microfluidic device was fabricated using the same protocol as previously described (Shojaeian \& Hardt, 2020). Briefly, a SU-8 photoresist on a silicon wafer was microstructured using ultraviolet (UV) lithography, after which a standard soft lithography protocol was applied. The resulting microstructured polydimethylsiloxane (PDMS) substrate was then bonded to a glass slide covered with the thin PDMS layer, yielding a microchannel architecture exclusively with PDMS walls. More information on device fabrication can be found in the supplementary information.

The experimental procedures are very similar to those previously reported (Shojaeian \& Hardt, 2020). Essentially, the main channel of the device is filled with silicone oil, the side channels with an aqueous salt solution. By applying a voltage pulse, a droplet is pinched off from the narrower side channel. The diameters of the droplets produced that way range between 2 and $16 \mu \mathrm{m}$. Subsequently, a DC electric field is applied between the side channels, upon which the droplet performs a reciprocating motion between the two liquid-liquid interfaces (in the following referred to as menisci). The droplet motion is imaged using an inverted microscope in brightfield mode (Nikon Eclipse Ti) and a high-speed camera (Redlake Motion Pro Series Y). More information on the experimental procedures can be found in the supplementary information. The working fluids are water at different salinities through addition of $\mathrm{NaCl}$ and $1000 \mathrm{cSt}$ silicone oil AP (Sigma-Aldrich). A table with the most important properties of the liquids used can be found in the supplementary information.

\section{Results and Discussion}

Different modes of droplet manipulation are possible with our microfluidic device. The standard mode is the reciprocating motion of droplets between the side channels, that can be used to introduce reagents into a droplet from one side channel and subtract reaction products from the opposing channel (Shojaeian \& Hardt, 2020). By switching off the electric field, the droplet can be fixed at a position between the side channels, while the exchange with the reservoirs is suspended. Most notably, the partial coalescence mode that was previously observed for much larger droplets (Hamlin et al., 2012) also occurs for our micron-scale droplets. More specifically, for a salt concentration inside the droplet below a threshold 
(a)

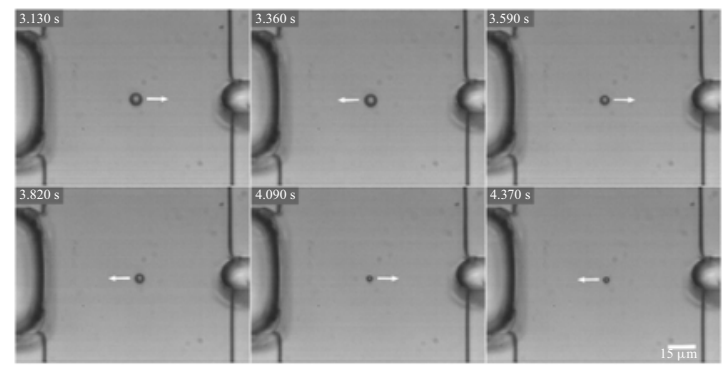

(b)

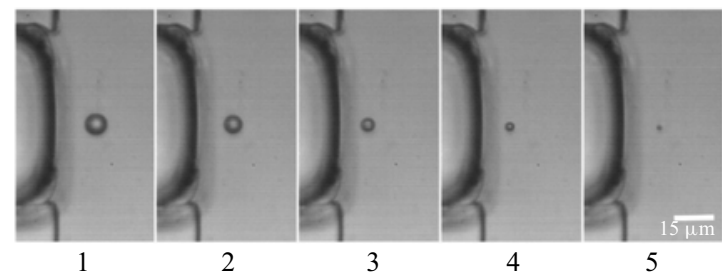

(c)

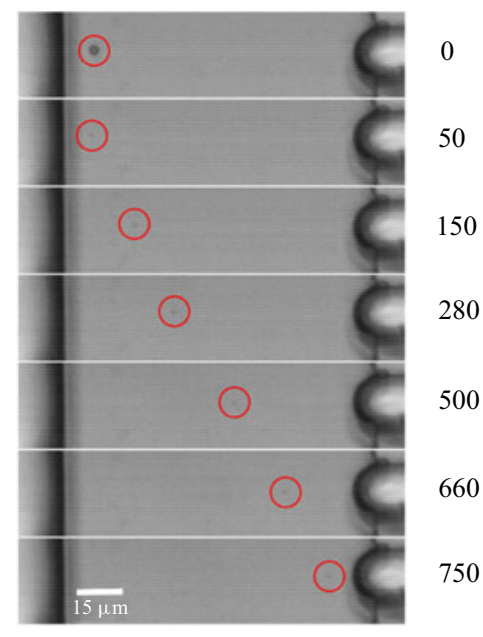

Figure 2. (a) Droplet reciprocating between the two menisci, undergoing partial coalescence at the left meniscus. The salt concentration is $0.17 \mathrm{mM}$ and a voltage of $200 \mathrm{~V}$ was applied between the menisci. (b) Snapshots of consecutive cycles, showing the same droplet before getting in contact with the left meniscus. (c) A droplet with a diameter of approximately $800 \mathrm{~nm}$ that has been formed by partial coalescence and travels from left to right. The numbers indicate the time in milliseconds, and the red circle encloses the droplet. The parameters in panels $b$ and $c$ are the same as in panel $a$.

value (in our case approx. $0.17 \mathrm{mM}$ ), a droplet may bounce back without volume loss for large enough values of the electric field strength, or it may experience a volume loss (partial coalescence) for smaller electric fields. This is demonstrated in Figure $2 a$. In this panel, and also in the other panels of this figure, the region of interest is rotated by $90^{\circ}$ relative to Figure 1 . Figure $2 a$ shows snapshots from subsequent cycles of a droplet reciprocating between the two side channels, where the direction of motion is indicated by arrows. Since the electric field lines mainly extend between the two menisci, the field strength at the right meniscus is higher than that at the left meniscus. As a result, the conditions at the right meniscus are such that a droplet bounces off from that meniscus, while it undergoes partial coalescence at the left meniscus. This process is visible in the supplementary movies.

Partial coalescence gives the opportunity to reduce the diameter of droplets to the submicron scale. Figure $2 b$ shows subsequent cycles from a reciprocating droplet motion shortly before undergoing partial coalescence with the left meniscus. The diameters of the droplets shown in the successive frames are 8.3, 6.9, 5.1, 3.4 and $1.6 \mu \mathrm{m}$. Our experiments do not give any indication that the cascade of droplet volume reduction comes to an end. However, due to the resolution limit of the optics, from a certain point on the droplets can no longer be imaged faithfully, but rather appear as a tiny grey patch. This is exemplified in Figure $2 c$. The figure shows a droplet experiencing a dramatic volume loss after partial coalescence with the left meniscus. The first frame shows the droplet before partial coalescence with the left meniscus. The daughter droplet, marked by the red circle, travels from left to right and has a diameter of approximately $800 \mathrm{~nm}$. To estimate the size of such small droplets, a Gaussian function was fitted to the grey-scale values of the pixels around the position of a droplet. The full width at half-maximum was taken as an estimate for the droplet diameter. The smallest droplets that could be identified based on this method have a diameter of approximately $400 \mathrm{~nm}$. However, the submicron diameters reported here should be taken with a grain of salt, since the Abbé resolution limit of the employed imaging system is approximately $600 \mathrm{~nm}$. This means that the apparent droplet diameters are expected to be larger than the true diameters, since diffraction tends to broaden the grey-scale distributions. We speculate that the 
cascade of partial coalescence events continues for even smaller droplets, but these could not be detected using the available imaging system.

One key question is what determines the size of the daughter droplet for a given size of the parent droplet. When fixing the size of the parent droplet and the voltage between the side channels, there is still some fluctuation in the size of the daughter droplet. There are a few reports on the factors influencing the partial electrocoalescence of charged droplets with an oil-water interface, albeit for much larger droplets than considered in this work. (Hamlin et al. 2012) performed experiments from which they deduced that

$$
\frac{a}{a_{0}}=K\left(\frac{q E}{\gamma a_{0}}\right)^{\alpha},
$$

where $q$ is the charge of the daughter droplet, $E$ is the electric field strength, $\gamma$ is the interfacial tension, $a$ is the radius of the daughter droplet and $a_{0}$ that of the parent droplet. For the scaling exponent they obtained $\alpha=1 / 2$ and for the pre-factor $K=0.85$. This is contrasted by the experiments reported by (Anand et al. 2020), who determined a scaling exponent $\alpha=3 / 2$.

To study which factors influence the size of the daughter droplet, we have performed a number of experiments with varying parent droplet size, salt concentration and applied voltage. In previous studies only the charge of the daughter droplet was considered, but it is not a priori clear that the charge of the parent droplet should not play an equally important role in the partial coalescence process. Therefore, we have determined the droplet charge before and after partial coalescence. Details are given in the supplementary material. Figure 3 shows the effects of the electric field and salt concentration on the daughter droplet size for droplets with the initial sizes of 7 and $11 \mu \mathrm{m}$. The ratio of daughter and parent droplet sizes is plotted as a function of the square root of the electric Bond number $\left(q E / \gamma a_{0}\right)^{1 / 2}$. This parameter is largely analogous to the parameter used in (Hamlin et al. 2012), with the difference that, in our case, $q$ is the arithmetic average of the daughter and parent droplet charges; $E$ denotes the electric field, which, however, only enters the equations in form of the product $q E$. This product was determined based on the procedure described in the supplementary material. Figure 3 indicates a similarity relationship, i.e. to a good approximation the experimental data depend linearly on the square root of the electric Bond number. In total, the scaling relationship is given by

$$
\frac{a}{a_{0}}=0.978\left(\frac{q E}{\gamma a_{0}}\right)^{1 / 2} \theta\left[\left(\frac{q E}{\gamma a_{0}}\right)^{1 / 2}-0.515\right],
$$

where $\theta$ is the Heaviside function and the numerical factors were determined based on a least-squares fit. A major difference to the results obtained for much larger droplets by Hamlin et al. lies in the fact that only above a critical value of the scaling parameter can partial coalescence occur. Translated to the charge of the droplet, this means that a minimum charge is required for partial coalescence, below that only full coalescence will occur. Equation (2) also allows us to check the results obtained for the size of droplets that are so small that they only appear as faint spots (cf. Figure $2 c$ ). From the velocity of these spots the droplet charge can be determined. The prediction of the scaling relationship is in good agreement with the Gaussian fits to the grey-scale distribution.

Via equation (2), the charge of a droplet is a key factor determining its volume loss upon partial coalescence. To study how the droplet charge evolves when a droplet reciprocates between the two menisci, we observed droplets over a large number of cycles. Figure 4 shows results for two different droplets obtained for a salt concentration of $0.17 \mathrm{mM}$ and a voltage of $250 \mathrm{~V}$. Panel a shows the droplet diameter and panel $\mathrm{b}$ the ratio $q E / a$, both as a function of cycle number. The quantities $q$ and $a$ are the droplet charge and radius measured right after partial coalescence with the meniscus at the larger side channel, respectively; $q E / a$ defines the scale of the electrostatic stress on the liquid-liquid interface, in the sense that dividing this ratio by $a$ yields a characteristic value of the stress. It can be seen that the droplets perform a large number of cycles upon which their diameter reduces gradually. After a critical diameter is reached, a rapid volume loss is observed. This transition corresponds to a transition in $q E / a$, 


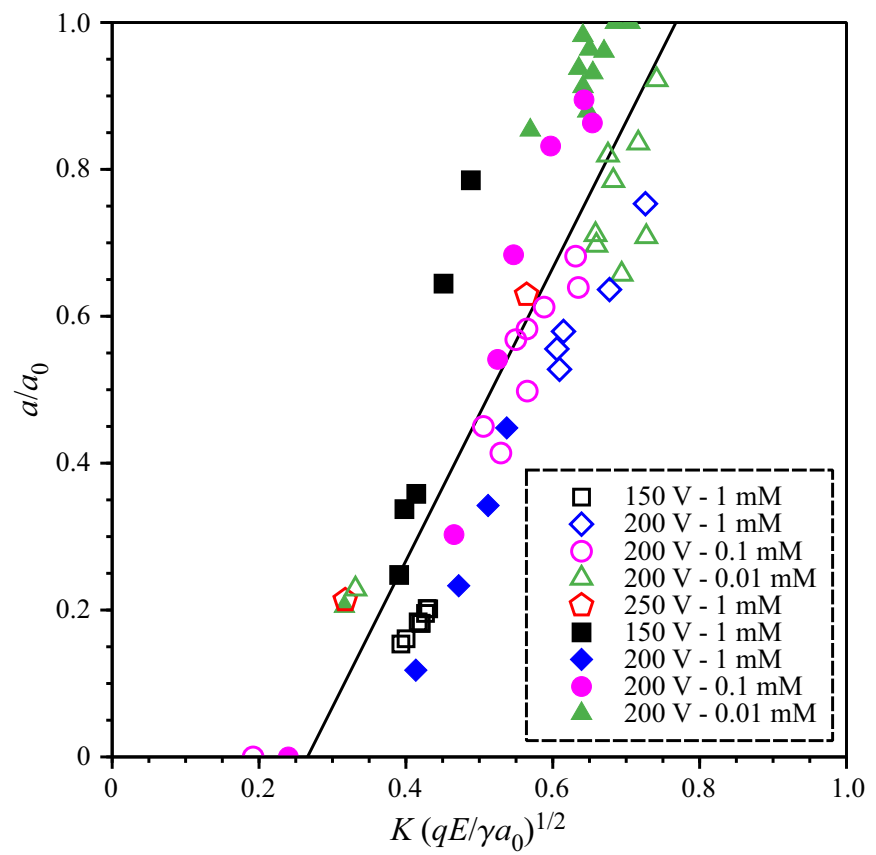

Figure 3. Dimensionless radius of the daughter droplet, plotted as a function of the square root of the electric Bond number. Experiments with different parent droplet diameters, salt concentrations and applied voltages were performed. The results for a parent droplet diameter of $7 \mu \mathrm{m}$ are represented as open symbols, those for $11 \mu \mathrm{m}$ as full symbols. The droplet charge could not be controlled in the experiments but was determined by measuring the droplet velocity.

which remains approximately constant before the critical diameter is reached and suddenly decreases above the critical diameter. Since the electric field was constant in these experiments, this means that, up to the critical diameter, $q / a$ remains approximately constant. Note that the values of $q E / a$ are of the same order of magnitude as the interfacial tension between the two liquids $\left(\sim 35 \mathrm{mN} \mathrm{m}^{-1}\right)$, which is an indication that the partial coalescence could be governed by a balance between interfacial tension and electrostatic forces. When the voltage is increased to $300 \mathrm{~V}$, qualitatively the same behaviour is observed as in Figure $4 a$, with a slightly reduced critical diameter (see supplementary information). If the voltage is too low (for the experiments shown in Figure $4 a, \sim 150 \mathrm{~V}$ ), a critical diameter below which the droplet size rapidly decreases is not observed. The critical diameter is independent of the initial droplet size at the start of the reciprocating motion, where we consider initial diameters larger than the critical diameter. The gradual decrease in droplet diameter visible in Figure $4 a$ can be utilized to consecutively withdraw minute samples from small droplets.

The discussion of the preceding paragraphs, especially the dependence on the electric Bond number $q E / \gamma a_{0}$, gives some vague hints to the physics underlying the partial coalescence of these micron-sized drops. While we emphasize that the purpose of this manuscript is not to discuss the flow physics in any detail, we wish to briefly comment on the underlying mechanisms. The physics of partial coalescence of millimetre-sized droplets has been studied in some detail (see, for example, Hamlin et al., 2012; Li et al., 2020, 2021). Compared with that, the droplets considered here have a diameter that is approximately three orders of magnitude smaller, implying that the corresponding dimensionless groups differ vastly as well. This excludes transferring the insights from larger scales to the smaller scales considered here.

We are only aware of one study that discusses the partial coalescence of micron-sized droplets under an electric field (Pillai et al., 2017). In this numerical study, equal properties of the droplet liquid and the oil phase were assumed, a marked difference to our studies where the oil had a viscosity more than three 
(a)

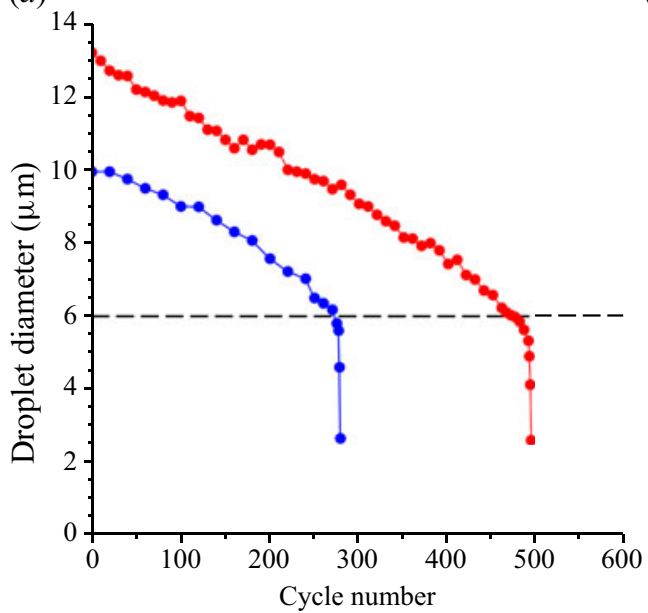

(b)

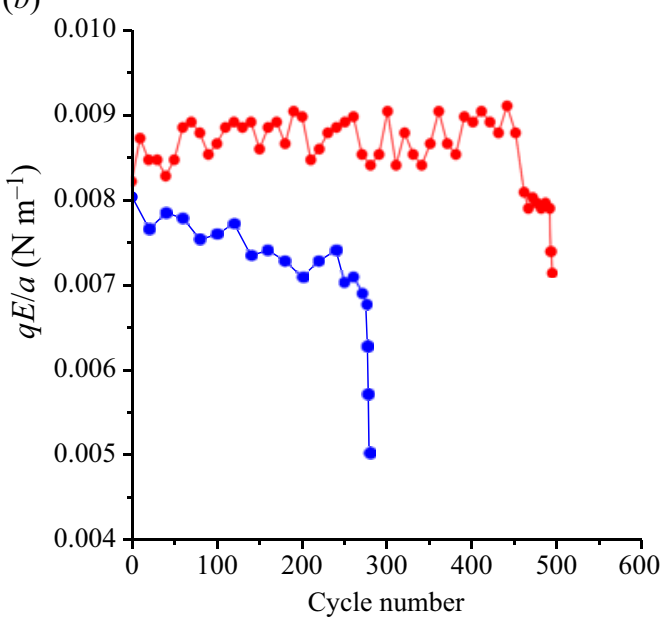

Figure 4. (a) Evolution of the diameter of two different droplets over a large number of cycles. The horizontal line indicates the approximate value of the critical diameter and serves as a guide to the eye. (b) Corresponding evolution of the parameter $q E / a$. The salt concentration was $0.17 \mathrm{mM}$ and the voltage $250 \mathrm{~V}$.

orders of magnitude higher than the droplet. Still, there are some similarities in the results. Roughly, in (Pillai et al. 2017), the partial coalescence mechanism is described as a competition between electrostatic and capillary forces. When a droplet approaches the meniscus, it is already polarized by the electric field, where the part of the droplet that faces the meniscus (the "south pole") carries charges of a sign opposite to that of the charges at the meniscus. After the droplet gets in touch with the meniscus, charge exchange happens, but most of the charges at the 'north pole' of the droplet remain there, inducing a force of the order of $q E$ that pulls the droplet away from the meniscus. This force is counteracted by the capillary force $\gamma a_{0}$ that pushes the droplet towards the meniscus. It is that balance that explains the emergence of the electric Bond number $q E / \gamma a_{0}$. (Pillai et al. 2017) and (Hamlin et al. 2012) agree as far as the relevance of this parameter is concerned, but they report different scaling exponents. Our scaling exponent agrees with that of (Hamlin et al. 2012), but an important difference to both papers is that in our case $q$ is the average between daughter and parent droplet charge, whereas both works referred to define $q$ as the daughter droplet charge. Our choice of the electric Bond number could hint to the fact that during the partial coalescence process charge is continuously transferred to the droplet, which makes a description only in terms of the daughter droplet charge questionable. Apart from that, more work is definitely needed to understand the physics of partial coalescence on such small scales. For example, what comes as a surprise considering the high oil viscosity is the fact that the viscosity does not enter Equation (2), at first sight suggesting that viscous forces do not play a role. Of course, the viscosity may be hidden in the numerical parameters appearing in this equation. Unfortunately, our attempts to study the influence of viscosity were unsuccessful, leaving this issue to be settled by future studies. The same type of silicone oil is also available with a viscosity of $100 \mathrm{cSt}$. It turned out that, with the lower viscosity silicone oil, upon application of an electric field, the menisci became unstable and the water phase protruded into the main channel. We speculate that surface-active contaminants are responsible for this failure mode.

\section{Summary, Conclusions and Outlook}

We demonstrated a scheme for manipulating droplets with volumes down to the sub-femtolitre range that is based on partial coalescence with a liquid reservoir under an electric field. Partial coalescence 
occurs when aqueous droplets with a not too high salt concentration reciprocate in an oil phase between two liquid-liquid menisci. The smallest droplets processed that way had a diameter of approximately $400 \mathrm{~nm}$. The experimental data on the droplet diameters before and after partial coalescence can be collapsed to a single curve when expressing the diameter ratio as a function of the ratio between the electrostatic stress acting on a droplet and the interfacial tension. This is an indication that the physics of partial coalescence is governed by a balance between the electrostatic stress and interfacial tension. When letting droplets reciprocate between the two menisci over a large number of cycles, first the droplet volume reduces rather gradually until a critical diameter is reached, after which the droplets shrink rapidly.

The scheme allows withdrawing minute samples from small droplets and could prove helpful in various applications where droplets are used as tiny reaction spaces or when the goal is to tailor the size of individual droplets. The process can be controlled by the applied electric field. When the field is switched off, a droplet has no exchange with the reservoirs. The exchange can be initiated by switching on the electric field. For large values of the field strength, a droplet can diffusively exchange dissolved species with the reservoirs without experiencing any volume loss (Shojaeian \& Hardt, 2020). For reduced field-strength values, this transitions into the partial coalescence mode described here. In this way, the electric field serves as a control parameter to control the interaction of tiny droplets with liquid reservoirs. On the fundamental side, there is a need of better understanding the physical mechanisms behind the partial coalescence of micron-sized droplets under electric fields. Also, it would be very interesting to explore the limits of the partial coalescence process. With the available experimental equipment, droplets with diameters below approximately $400 \mathrm{~nm}$ could no longer be detected, but it could well be that partial coalescence even occurs for nanodroplets.

Acknowledgements. We thank T. Meckel, TU Darmstadt, for helpful discussions on microscopy techniques, K.-D. Voss, TU Darmstadt, for his assistance in microfabrication, and S. Dehe, TU Darmstadt, for his assistance in composing the graphical abstract.

Competing Interests. The authors declare no conflicts of interest.

Author Contributions. Conceptualization: M.S.; S.H. Methodology: M.S; S.H. Data curation: M.S. Data visualisation: M.S. Writing original draft: S.H. All authors approved the final submitted draft.

Data Availability Statement. The research data related to this work are available at https://tudatalib.ulb.tu-darmstadt.de/handle/ tudatalib/2847.

Ethical Standards. The research meets all ethical guidelines, including adherence to the legal requirements of the study country.

Supplementary Material and Movies. Supplementary material and movies are available at https://doi.org/10.1017/flo.2021.12.

\section{References}

Abate, A. R., Hung, T., Marya, P., Agresti, J. J., \& Weitz, D. A. (2010). High-throughput injection with microfluidics using picoinjectors using picoinjectors. Proceedings of the National Academy of Sciences of the United States of America, 107(45), 19163-19166. doi:10.1073/pnas.1006888107

Actis, P. (2018). Sampling from single cells. Small Methods, 2(3), 1700300. doi:10.1002/smtd.201700300

Allan, R. S., \& Mason, S. G. (1961). Effects of electric fields on coalescence in liquid+liquid systems. Transactions of the Faraday Society, 57, 2027-2040.

Anand, V., Juvekar, V. A., \& Thaokar, R. M. (2020). Coalescence, partial coalescence, and noncoalescence of an aqueous drop at an oil-water interface under an electric field. Langmuir, 36(21), 6051-6060. doi:10.1021/acs.langmuir.9b03969

Anna, S. L., \& Mayer, H. C. (2006). Microscale tipstreaming in a microfluidic flow focusing device. Physics of Fluids, $18,12$. doi:10.1063/1.2397023

Batchelor-McAuley, C., Little, C. A., Sokolov, S. V., Katelhön, E., Zampardi, G., \& Compton, R. G. (2016). Fluorescence monitored voltammetry of single attoliter droplets. Analytical Chemistry, 88(22), 11213-11221. doi:10.1021/acs.analchem.6b03524

Beneyton, T., Krafft, D., Bednarz, C., Kleineberg, C., Woelfer, C., Ivanov, I., ... Baret, J. C. (2018). Out-ofequilibrium microcompartments for the bottom-up integration of metabolic functions. Nature Communications, 9(1), 1-10. doi:10.1038/s41467-018-04825-1

Charles, G. E., \& Mason, S. G. (1960a). The coalescence of liquid drops with flat liquid/liquid interfaces. Journal of Colloid Science, 15, 236-267. 
Charles, G. E., \& Mason, S. G. (1960b). The mechanism of partial coalescence of liquid drops at liquid/liquid interfaces. Journal of Colloid Science, 15, 105-122.

Delmas, T., Piraux, H., Couffin, A. C., Texier, I., Vinet, F., Poulin, P., . . Bibette, J. (2011). How to prepare and stabilize very small nanoemulsions. Langmuir, 27(5), 1683-1692. doi:10.1021/la104221q

dos Santos, E. C., Belluati, A., Necula, D., Scherrer, D., Meyer, C. E., Wehr, R. P., . . Meier, W. (2020). Combinatorial strategy for studying biochemical pathways in double emulsion templated cell-sized compartments. Advanced Materials, 32(48), 1-13. doi:10.1002/adma.202004804

Fanalista, F., Birnie, A., Maan, R., Burla, F., Charles, K., Pawlik, G., .. Dekker, C. (2019). Shape and size control of artificial cells for bottom-up biology. ACS Nano, 13(5), 5439-5450. doi:10.1021/acsnano.9b00220

Fernández de la Mora, J. (2007). The fluid dynamics of Taylor Cones. Annual Review of Fluid Mechanics, 39, $217-243$. doi:10.1146/annurev.fluid.39.050905.110159

Gach, P. C., Iwai, K., Kim, P. W., Hillson, N. J., \& Singh, A. K. (2017). Droplet microfluidics for synthetic biology. Lab on a Chip, 17(20), 3388-3400. doi:10.1039/c71c00576h

Göpfrich, K., Platzman, I., \& Spatz, J. P. (2018). Mastering complexity: towards bottom-up construction of multifunctional eukaryotic synthetic cells. Trends in Biotechnology, 36(9), 938-951. doi:10.1016/j.tibtech.2018.03.008

Håkansson, A. (2019). Emulsion formation by homogenization: current understanding and future perspectives. Annual Review of Food Science and Technology, 10, 239-258. doi:10.1146/annurev-food-032818-121501

Haller, B., Göpfrich, K., Schröter, M., Janiesch, J. W., Platzman, I., \& Spatz, J. P. (2018). Charge-controlled microfluidic formation of lipid-based single- and multicompartment systems. Lab on a Chip, 18(17), 2665-2674. doi:10.1039/c81c00582f

Hamlin, B. S., Creasey, J. C., \& Ristenpart, W. D. (2012). Electrically tunable partial coalescence of oppositely charged drops. Physical Review Letters, 109(9), 1-5. doi:10.1103/PhysRevLett.109.094501

Kim, B. K., Kim, J., \& Bard, A. J. (2015). Electrochemistry of a single attoliter emulsion droplet in collisions. Journal of the American Chemical Society, 137(6), 2343-2349. doi:10.1021/ja512065n

Li, B., Dou, X., Yu, K., Huang, Y., Zhang, W., Xu, H., ... Wang, J. (2021). Coalescence dynamic response of an aqueous droplet at an oil-water interface under a steady electric field. International Journal of Multiphase Flow, $139,103628$. doi:10.1016/j.ijmultiphaseflow.2021.103628

Li, B., Wang, Z., Vivacqua, V., Ghadiri, M., Wang, J., Zhang, W., .. . Wang, Z. (2020). Drop-interface electrocoalescence mode transition under a direct current electric field. Chemical Engineering Science, 213, 115360. doi:10.1016/j.ces.2019.115360

Malloggi, F., Pannacci, N., Attia, R., Monti, F., Mary, P., Willaime, H., . . Poncet, P. (2010). Monodisperse colloids synthesized with nanofluidic technology. Langmuir, 26(4), 2369-2373. doi:10.1021/la9028047

Marín, A. G., Campo-Cortés, F., \& Gordillo, J. M. (2009). Generation of micron-sized drops and bubbles through viscous coflows. Colloids and Surfaces A: Physicochemical and Engineering Aspects, 344(1-3), 2-7. doi:10.1016/j.colsurfa.2008.09.033

Pillai, R., Berry, J. D., Harvie, D. J. E., \& Davidson, M. R. (2017). Electrophoretically mediated partial coalescence of a charged microdrop. Chemical Engineering Science, 169, 273-283. doi:10.1016/j.ces.2016.07.022

Ristenpart, W. D., Bird, J. C., Belmonte, A., Dollar, F., \& Stone, H. A. (2009). Non-coalescence of oppositely charged drops. Nature, 461(7262), 377-380. doi:10.1038/nature08294

Shojaeian, M., \& Hardt, S. (2020). Mass transfer via femtoliter droplets in ping-pong mode. Physical Review Applied, $13(1), 1$. doi:10.1103/PhysRevApplied.13.014015

Shojaeian, M., Lehr, F. X., Göringer, H. U., \& Hardt, S. (2019). On-demand production of femtoliter drops in microchannels and their use as biological reaction compartments. Analytical Chemistry, 91(5), 3484-3491. doi:10.1021/acs.analchem.8b05063

Song, H., Li, H. W., Munson, M. S., Ha, T. G. V., \& Ismagilov, R. F. (2006). On-chip titration of an anticoagulant argatroban and determination of the clotting time within whole blood or plasma using a plug-based microfluidic system. Analytical Chemistry, 78(14), 4839-4849. doi:10.1021/ac0601718

Suryo, R., \& Basaran, O. A. (2006). Tip streaming from a liquid drop forming from a tube in a co-flowing outer fluid. Physics of Fluids, 18, 8. doi:10.1063/1.2335621

Toprakcioglu, Z., Challa, P. K., Morse, D. B., \& Knowles, T. (2020). Attoliter protein nanogels from droplet nanofluidics for intracellular delivery. Science Advances, 6(6), 1-9. doi:10.1126/sciadv.aay7952

Van Nies, P., Westerlaken, I., Blanken, D., Salas, M., Mencía, M., \& Danelon, C. (2018). Self-replication of DNA by its encoded proteins in liposome-based synthetic cells. Nature Communications, 9(1), 1-12. doi:10.1038/s41467-018-03926-1

Vladisavljević, G. T. (2019). Preparation of microemulsions and nanoemulsions by membrane emulsification. Colloids and Surfaces A: Physicochemical and Engineering Aspects, 579, 123709. doi:10.1016/j.colsurfa.2019.123709

Wang, X., Du, H., Wang, Z., Mu, W., \& Han, X. (2020). Versatile phospholipid assemblies for functional synthetic cells and artificial tissues. Advanced Materials, 33(6), 2002635. doi:10.1002/adma.202002635

Wang, Y., Shan, X., Cui, F., Li, J., Wang, S., \& Tao, N. (2015). Electrochemical reactions in subfemtoliter-droplets studied with plasmonics-based electrochemical current microscopy. Analytical Chemistry, 87(1), 494-498. doi:10.1021/ac5036692

Weiss, M., Frohnmayer, J. P., Benk, L. T., Haller, B., Janiesch, J. W., Heitkamp, T., . . Spatz, J. P. (2018). Sequential bottom-up assembly of mechanically stabilized synthetic cells by microfluidics. Nature Materials, 17(1), 89-95. doi:10.1038/NMAT5005

Zeng, S., Pan, X., Zhang, Q., Lin, B., \& Qin, J. (2011). Electrical control of individual droplet breaking and droplet contents extraction. Analytical Chemistry, 83(6), 2083-2089. doi:10.1021/ac1028775

Cite this article: Shojaeian, M., \& Hardt, S. (2021). Manipulation of single sub-femtolitre droplets via partial coalescence in a direct-current 